\title{
Asymptotic Method of Krylov-Bogoliubov-Mitropolskii for Fifth Order Critically Damped Nonlinear Systems
}

\author{
Md. Nazrul Islam¹, Md. Mahafujur Rahaman ${ }^{2, ~ *, ~ M . ~ A b u l ~ K a w s e r ~}{ }^{1}$ \\ ${ }^{1}$ Department of Mathematics, Islamic University, Kushtia, Bangladesh \\ ${ }^{2}$ Department of Computer Science \& Engineering, Z. H. Sikder University of Science \& Technology, Shariatpur, Bangladesh
}

Email address:

nazru1001bd@gmail.com (Md. N. Islam),mahfuz0809@gmail.com (Md. M. Rahaman), kawserma@yahoo.com (M. A. Kawser)

\section{To cite this article:}

Md. Nazrul Islam, Md. Mahafujur Rahaman, M. Abul Kawser. Asymptotic Method of Krylov-Bogoliubov-Mitropolskii for Fifth Order Critically Damped Nonlinear Systems. Applied and Computational Mathematics. Vol. 4, No. 6, 2015, pp. 387-395.

doi: $10.11648 /$ j.acm. 20150406.11

\begin{abstract}
In oscillatory problems, the method of Krylov-Bogoliubov-Mitropolskii (KBM) is one of the most used techniques to obtain analytical approximate solution of nonlinear systems with a small non-linearity. This article modifies the KBM method to examine the solutions of fifth order critically damped nonlinear systems with four pairwise equal eigenvalues and one distinct eigenvalue, in which the latter eigenvalue is much larger than the former four pairwise eigenvalues. This paper suggests that the results obtained in this study correspond accurately to the numerical solutions obtained by the fourth order Runge-Kutta method. This paper, therefore, concludes that the modified KBM method provides highly accurate results, which can be applied for different kinds of nonlinear differential systems.
\end{abstract}

Keywords: KBM, Asymptotic Method, Critically Damped System, Nonlinearity, Runge-Kutta Method, Eigenvalues

\section{Introduction}

In oscillatory problems, the method of KrylovBogoliubov-Mitropolskii (KBM) $[1,2]$ is particularly convenient, and is the vastly used technique to obtain analytical approximate solution of nonlinear systems with a small non-linearity. The method was, in fact, developed by Krylov and Bogoliubov [2] for obtaining periodic solutions, which was amplified and justified by Bogoliubov and Mitropolskii [1], and later extended by Popov [3] and Meldelson [4] for damped nonlinear oscillations. Murty [5] developed a unified KBM method for solving second-order nonlinear systems. Sattar [6] studied a third-order overdamped nonlinear system. Bojadziev [7] studied the damped oscillations modeled by a three dimensional nonlinear system. Shamsul and Sattar [8] developed a method for third order critically-damped nonlinear equations. Rokibul and Akbar [9] investigated a new solution of third order more critically damped nonlinear systems. Shamsul and Sattar [10] presented a unified KBM method for solving third-order nonlinear systems. Akbar et al. [11] presented a method for solving the fourth-order over-damped nonlinear systems. Rokibul et al. [12] presented a new technique for fourth order critically damped nonlinear systems with some conditions.
Rahaman and Rahman [13] suggested analytical approximate solutions of fifth order more critically damped systems in the case of smaller triply repeated roots. Rahaman and Kawser [14] also proposed asymptotic solutions of fifth order critically damped nonlinear systems with pairwise equal eigenvalues and another is distinct.

The aim of this article is to obtain the analytical approximate solutions of fifth order critically damped nonlinear systems by extending the KBM method. In this study, it is suggested that the results obtained by the perturbation solution have been compared with those obtained by the fourth order Runge-Kutta method.

\section{The Method}

We are going to propose a perturbation technique to solve fifth order non-linear differential systems of the form

$$
x^{(v)}+k_{1} x^{(i v)}+k_{2} \dddot{x}+k_{3} \ddot{x}+k_{4} \dot{x}+k_{5} x=-\varepsilon f\left(x, \dot{x}, \ddot{x}, \dddot{x}, x^{(i v)}\right)
$$

where $\mathrm{x}^{(\mathrm{v})}$ and $\mathrm{x}^{(\mathrm{iv})}$ stand for the fifth and fourth derivatives respectively, and over dots are used for the first, second and third derivatives of $x$ with respect to $t$; $\mathrm{k}_{1}, \mathrm{k}_{2}, \mathrm{k}_{3}, \mathrm{k}_{4}, \mathrm{k}_{5}$ are constants, $\varepsilon$ is a sufficiently small 
parameter and $f\left(x, \dot{x}, \ddot{x}, \dddot{x}, x^{(i v)}\right)$ is the given nonlinear function. As the unperturbed equation (1) has five real negative eigenvalues, where four eigenvalues are pairwise equal and the other one is distinct. Here, the distinct eigenvalue is much larger than the pairwise equal eigenvalues. Now, suppose that the eigenvalues are $-\lambda,-\lambda,-\mu,-\mu,-v$.

When $\varepsilon=0$, the equation (1) becomes linear and the solution of the corresponding linear equation becomes

$$
x(t, 0)=\left(a_{0}+b_{0} t\right) e^{-\lambda t}+\left(c_{0}+d_{0} t\right) e^{-\mu t}+h_{0} e^{-v t}
$$

where $\mathrm{a}_{0}, \mathrm{~b}_{0}, \mathrm{c}_{0}, \mathrm{~d}_{0}$ and $\mathrm{h}_{0}$ are constants of integration.

When $\varepsilon \neq 0$ following Shamsul [15], an asymptotic solution of the equation (1) is sought in the form

$$
\begin{aligned}
\mathrm{x}(\mathrm{t}, \varepsilon) & =(\mathrm{a}+\mathrm{bt}) \mathrm{e}^{-\lambda \mathrm{t}}+(\mathrm{c}+\mathrm{dt}) \mathrm{e}^{-\mu \mathrm{t}}+\mathrm{he}^{-v \mathrm{t}} \\
& +\varepsilon \mathrm{u}_{1}(\mathrm{a}, \mathrm{b}, \mathrm{c}, \mathrm{d}, \mathrm{h}, \mathrm{t})+\ldots
\end{aligned}
$$

where $a, b, c, d$ and $h$ are the functions of $t$ and they satisfy the first order differential equations

$$
\begin{gathered}
\dot{\mathrm{a}}=\varepsilon \mathrm{A}_{1}(\mathrm{a}, \mathrm{b}, \mathrm{c}, \mathrm{d}, \mathrm{h}, \mathrm{t})+\ldots \dot{\mathrm{b}}=\varepsilon \mathrm{B}_{1}(\mathrm{a}, \mathrm{b}, \mathrm{c}, \mathrm{d}, \mathrm{h}, \mathrm{t})+\ldots \\
\dot{\mathrm{c}}=\varepsilon \mathrm{C}_{1}(\mathrm{a}, \mathrm{b}, \mathrm{c}, \mathrm{d}, \mathrm{h}, \mathrm{t})+\ldots \\
\dot{\mathrm{d}}=\varepsilon \mathrm{D}_{1}(\mathrm{a}, \mathrm{b}, \mathrm{c}, \mathrm{d}, \mathrm{h}, \mathrm{t})+\ldots \dot{\mathrm{h}}=\varepsilon \mathrm{H}_{1}(\mathrm{a}, \mathrm{b}, \mathrm{c}, \mathrm{d}, \mathrm{h}, \mathrm{t})+\ldots
\end{gathered}
$$

Now differentiating (3) five times with respect to $t$, substituting the value of $x$ and the derivatives $\mathrm{x}^{(\mathrm{v})}, \mathrm{x}^{(\mathrm{iv})}, \dddot{\mathrm{x}}, \ddot{\mathrm{x}}, \dot{\mathrm{x}}$ in the original equation (1) utilizing the relations presented in (4) and finally extracting the coefficients of $\varepsilon$, we obtain

$$
\begin{aligned}
& \mathrm{e}^{-\lambda t}(\mathrm{D}+\mu-\lambda)^{2}(\mathrm{D}+v-\lambda)\left(\begin{array}{c}
\frac{\partial \mathrm{A}_{1}}{\partial \mathrm{t}}+2 \mathrm{~B}_{1} \\
+\mathrm{t} \frac{\partial \mathrm{B}_{1}}{\partial \mathrm{t}}
\end{array}\right)+ \\
& \mathrm{e}^{-\mu t}(\mathrm{D}+\lambda-\mu)^{2}(\mathrm{D}+v-\mu)\left(\begin{array}{c}
\frac{\partial \mathrm{C}_{1}}{\partial \mathrm{t}}+2 \mathrm{D}_{1} \\
+\mathrm{t} \frac{\partial \mathrm{D}_{1}}{\partial \mathrm{t}}
\end{array}\right)+ \\
& \mathrm{e}^{-v \mathrm{t}}(\mathrm{D}+\lambda-v)^{2}(\mathrm{D}+\mu-v)^{2} \mathrm{H}_{1}+ \\
& (\mathrm{D}+\lambda)^{2}(\mathrm{D}+\mu)^{2}(\mathrm{D}+v) \mathrm{u}_{1}=-\mathrm{f}^{0}(\mathrm{a}, \mathrm{b}, \mathrm{c}, \mathrm{d}, \mathrm{h}, \mathrm{t})
\end{aligned}
$$

Where $f^{(0)}(a, b, c, d, h, t)=f\left(x, \dot{x}, \ddot{x}, \dddot{x}, x^{\text {iv }}\right)$

$$
\text { And } x(t, 0)=\left(a_{0}+b_{0} t\right) e^{-\lambda t}+\left(c_{0}+d_{0} t\right) e^{-\mu t}+h_{0} e^{-v t}
$$

We have expanded the function $\mathrm{f}^{(0)}$ in the Taylor's series (Sattar [16], Shamsul [17, 18], Shamsul and Sattar [8]) about the origin in power of $t$. Therefore, we obtain

$$
f^{(0)}=\sum_{q=0}^{\infty}\left\{t^{q} \sum_{i, j, k, l=0}^{\infty} F_{q, 1}(a, b, c, d, h) e^{-(i \lambda+j \mu+k v) t}\right\}
$$

$$
\begin{aligned}
& \mathrm{e}^{-\lambda \mathrm{t}}(\mathrm{D}+\mu-\lambda)^{2}(\mathrm{D}+v-\lambda)\left(\begin{array}{c}
\frac{\partial \mathrm{A}_{1}}{\partial \mathrm{t}}+2 \mathrm{~B}_{1} \\
+\mathrm{t} \frac{\partial \mathrm{B}_{1}}{\partial \mathrm{t}}
\end{array}\right)+ \\
& \mathrm{e}^{-\mu \mathrm{t}}(\mathrm{D}+\lambda-\mu)^{2}(\mathrm{D}+v-\mu)\left(\begin{array}{l}
\frac{\partial \mathrm{C}_{1}}{\partial \mathrm{t}}+ \\
2 \mathrm{D}_{1}+\mathrm{t} \frac{\partial \mathrm{D}_{1}}{\partial \mathrm{t}}
\end{array}\right)+ \\
& \mathrm{e}^{-v \mathrm{t}}(\mathrm{D}+\lambda-v)^{2}(\mathrm{D}+\mu-v)^{2} \mathrm{H}_{1}+ \\
& (\mathrm{D}+\lambda)^{2}(\mathrm{D}+\mu)^{2}(\mathrm{D}+v) \mathrm{u}_{1}= \\
& -\sum_{\mathrm{q}=0}^{\infty}\left\{\mathrm{t}^{\mathrm{q}} \sum_{\mathrm{i}, \mathrm{j}, \mathrm{k}=0}^{\infty}(\mathrm{a}, \mathrm{b}, \mathrm{c}, \mathrm{d}, \mathrm{h}) \mathrm{e}^{-(\mathrm{i} \lambda+\mathrm{j} \mu+\mathrm{kv}) \mathrm{t}}\right\}
\end{aligned}
$$

Following the KBM method, Murty and Deekshatulu [19], Sattar [16], Shamsul [18], and Shamsul and Sattar $[8,20]$ imposed the condition that $\mathrm{u}_{1}$ does not contain the fundamental terms (the solution (2) is called the generating solution and its terms are called the fundamental terms) of $\mathrm{f}^{(0)}$. Therefore, equation (7) can be separated for unknown functions $A_{1}, B_{1}, C_{1}, D_{1}, H_{1}$ and $u_{1}$ in the following way:

$$
\begin{aligned}
& \mathrm{e}^{-\lambda t}(\mathrm{D}+\mu-\lambda)^{2}(\mathrm{D}+v-\lambda)\left(\begin{array}{c}
\frac{\partial \mathrm{A}_{1}}{\partial \mathrm{t}}+2 \mathrm{~B}_{1} \\
+\mathrm{t} \frac{\partial \mathrm{B}_{1}}{\partial \mathrm{t}}
\end{array}\right)+ \\
& \mathrm{e}^{-\mu \mathrm{t}}(\mathrm{D}+\lambda-\mu)^{2}(\mathrm{D}+v-\mu)\left(\begin{array}{c}
\frac{\partial \mathrm{C}_{1}}{\partial \mathrm{t}}+2 \mathrm{D}_{1} \\
+\mathrm{t} \frac{\partial \mathrm{D}_{1}}{\partial \mathrm{t}}
\end{array}\right)+ \\
& \mathrm{e}^{-\mathrm{vt}}(\mathrm{D}+\lambda-v)^{2}(\mathrm{D}+\mu-v)^{2} \mathrm{H}_{1}= \\
& -\sum_{\mathrm{q}=0}^{1}\left\{\mathrm{t}^{\mathrm{q}} \sum_{\mathrm{i}, \mathrm{j}, \mathrm{k}, \mathrm{l}=0}^{\infty} \mathrm{F}_{\mathrm{q}, \mathrm{l}}(\mathrm{a}, \mathrm{b}, \mathrm{c}, \mathrm{d}, \mathrm{h}) \mathrm{e}^{-(\mathrm{i} \lambda+\mathrm{j} \mu+\mathrm{kv}) \mathrm{t}}\right\} \\
& (\mathrm{D}+\lambda)^{2}(\mathrm{D}+\mu)^{2}(\mathrm{D}+\mathrm{v}) \mathrm{u}_{1}= \\
& -\sum_{\mathrm{q}=2}^{\infty}\left\{\mathrm{t}^{\mathrm{q}} \sum_{\mathrm{i}, \mathrm{j}, \mathrm{k}, \mathrm{l}=0}^{\infty} \mathrm{F}_{\mathrm{q}, 1}(\mathrm{a}, \mathrm{b}, \mathrm{c}, \mathrm{d}, \mathrm{h}) \mathrm{e}^{-(\mathrm{i} \lambda+\mathrm{j} \mu+\mathrm{kv}) \mathrm{t}}\right\}
\end{aligned}
$$

Now equating the coefficients of $t^{0}, t^{1}$ from equation (8), we obtain

$$
\begin{gathered}
\mathrm{e}^{-\lambda \mathrm{t}}(\mathrm{D}+\mu-\lambda)^{2}(\mathrm{D}+v-\lambda)\left(\frac{\partial \mathrm{A}_{1}}{\partial \mathrm{t}}+2 \mathrm{~B}_{1}\right) \\
+\mathrm{e}^{-\mu \mathrm{t}}(\mathrm{D}+\lambda-\mu)^{2}(\mathrm{D}+v-\mu)\left(\frac{\partial \mathrm{C}_{1}}{\partial \mathrm{t}}+2 \mathrm{D}_{1}\right) \\
+\mathrm{e}^{-v \mathrm{t}}(\mathrm{D}+\lambda-v)^{2}(\mathrm{D}+\mu-v)^{2} \mathrm{H}_{1}=- \\
\sum_{\mathrm{i}, \mathrm{j}, \mathrm{k}, \mathrm{l}=0}^{\infty} \mathrm{F}_{0,1}(\mathrm{a}, \mathrm{b}, \mathrm{c}, \mathrm{d}, \mathrm{h}) \mathrm{e}^{-(\mathrm{i} \lambda+\mathrm{j} \mu+\mathrm{kv}) \mathrm{t}}
\end{gathered}
$$

Thus, using (6), the equation (5) becomes 


$$
\begin{gathered}
\mathrm{e}^{-\lambda \mathrm{t}}(\mathrm{D}+\mu-\lambda)^{2}(\mathrm{D}+\mathrm{v}-\lambda) \frac{\partial \mathrm{B}_{1}}{\partial \mathrm{t}}+\mathrm{e}^{-\mu \mathrm{t}} \\
(\mathrm{D}+\lambda-\mu)^{2}(\mathrm{D}+\mathrm{v}-\mu) \frac{\partial \mathrm{D}_{1}}{\partial \mathrm{t}}=- \\
\sum_{\mathrm{i}, \mathrm{j}, \mathrm{k}, \mathrm{l}=0}^{\infty} \mathrm{F}_{1,1}(\mathrm{a}, \mathrm{b}, \mathrm{c}, \mathrm{d}, \mathrm{h}) \mathrm{e}^{-(\mathrm{i} \lambda+\mathrm{j} \mu+\mathrm{k} v) \mathrm{t}}
\end{gathered}
$$

Here, we have only two equations (10) and (11) for determining the unknown functions $A_{1}, B_{1}, C_{1}, D_{1}$ and $H_{1}$ Thus, to obtain the unknown functions $A_{1}, B_{1}, C_{1}, D_{1}$ and $H_{1}$, we need to impose some conditions (Shamsul [18, 20, 21, 23]) between the eigenvalues. Different authors have imposed different conditions according to the behavior of the systems, such as Shamsul [21] imposed the condition

$$
\mathrm{i}_{1} \lambda_{1}+\mathrm{i}_{2} \lambda_{2}+\ldots+\mathrm{i}_{\mathrm{n}} \lambda_{\mathrm{n}} \leq\left(\mathrm{i}_{1}+\mathrm{i}_{1}+\ldots+\mathrm{i}_{1}\right)\left(\lambda_{1}+\lambda_{2}+\ldots+\lambda_{\mathrm{n}}\right)
$$

In this study, we have investigated solutions for the cases $\lambda \gg \mu$ and $\lambda<<v$. Therefore, we shall be able to separate the equation (11) for unknown functions $B_{1}$ and $D_{1}$; and solving them for $B_{1}$ and $D_{1}$ substituting the values of $B_{1}$ and $D_{1}$ into the equation (11) and applying the conditions $\lambda \gg>\mu$ and $\lambda<<v$, we can separate the equation (12) for three unknown functions $A_{1}, C_{1}$ and $H_{1}$; and solving them for $A_{1}$, $C_{1}$ and $H_{1}$. Since $\dot{\mathrm{a}}, \dot{\mathrm{b}}, \dot{\mathrm{c}}, \dot{\mathrm{d}}$ and $\dot{\mathrm{h}}$ are proportional to small parameter, they are slowly varying functions of time $t$, and for first approximate solution, we may consider them as constants in the right side. This assumption was first made by Murty and Deekshatulu [19]. Thus, the solutions of the equation (4) become

$$
\begin{aligned}
& a=a_{0}+\varepsilon \int_{0}^{t} A_{1}(a, b, c, d, h, t) d t \\
& b=b_{0}+\varepsilon \int_{0}^{t} B_{1}(a, b, c, d, h, t) d t \\
& c=c_{0}+\varepsilon \int_{0}^{t} C_{1}(a, b, c, d, h, t) d t \\
& d=d_{0}+\varepsilon \int_{0}^{t} D_{1}(a, b, c, d, h, t) d t \\
& h=h_{0}+\varepsilon \int_{0}^{t} H_{1}(a, b, c, d, h, t) d t
\end{aligned}
$$

Equation (9) is a non-homogeneous linear ordinary differential equation; therefore, it can be solved by the wellknown operator method. Substituting the values of $a, b, c, d$, $h$ and $\mathrm{u}_{1}$ in the equations (3), we shall get the complete solution of (1). Therefore, the determination of the first approximate solution is complete.

\section{Example}

As an example of the above method, we have considered the Duffing type equation of fifth order nonlinear differential system:

$$
x^{(v)}+k_{1} x^{(i v)}+k_{2} \dddot{x}+k_{3} \ddot{x}+k_{4} \dot{x}+k_{5} x=-\varepsilon x^{3}
$$

Comparing equation (13) and equation (1), we obtain $\mathrm{f}\left(\mathrm{x}, \dot{\mathrm{x}}, \ddot{\mathrm{x}}, \dddot{\mathrm{x}}, \mathrm{x}^{(\mathrm{iv})}\right)=\mathrm{x}^{3}$

Therefore,

$$
\begin{aligned}
& \mathrm{f}^{(0)}=\mathrm{a}^{3} \mathrm{e}^{-3 \lambda \mathrm{t}}+3 \mathrm{a}^{2} \mathrm{ce} \mathrm{e}^{-(2 \lambda+\mu) \mathrm{t}}+3 \mathrm{a}^{2} h \mathrm{e}^{-(2 \lambda+v) \mathrm{t}} \\
& +3 \mathrm{ac}^{2} \mathrm{e}^{-(\lambda+2 \mu) \mathrm{t}}+6 \mathrm{ache}^{-(\lambda+\mu+v) \mathrm{t}}+3 \mathrm{ah}^{2} \\
& \mathrm{e}^{-(\lambda+2 v) \mathrm{t}}+\mathrm{c}^{3} \mathrm{e}^{-3 \mu \mathrm{t}}+3 \mathrm{c}^{2} h \mathrm{e}^{-(2 \mu+v) \mathrm{t}}+3 \mathrm{ch}^{2} \\
& \mathrm{e}^{-(\mu+2 v) \mathrm{t}}+\mathrm{h}^{3} \mathrm{e}^{-3 v \mathrm{t}}+3 \mathrm{t}\left\{\mathrm{a}^{2} \mathrm{be}^{-3 \lambda \mathrm{t}}+2 \mathrm{abce} \mathrm{e}^{-(2 \lambda+\mu) \mathrm{t}}\right. \\
& +\mathrm{a}^{2} \mathrm{de}^{-(2 \lambda+\mu) \mathrm{t}}+2 \mathrm{abhe} \mathrm{e}^{-(2 \lambda+v) \mathrm{t}}+2 \mathrm{acde}^{-(\lambda+2 \mu) \mathrm{t}} \\
& +\mathrm{bc}^{2} \mathrm{e}^{-(\lambda+2 \mu) \mathrm{t}}+2 \mathrm{adhe}^{-(\lambda+\mu+v) \mathrm{t}}+2 \mathrm{bche}^{-(\lambda+\mu+v) \mathrm{t}} \\
& +\mathrm{bh}^{2} \mathrm{e}^{-(\lambda+2 v) \mathrm{t}}+\mathrm{c}^{2} \mathrm{de}^{-3 \mu \mathrm{t}}+2 \mathrm{cdh} \mathrm{e}^{-(2 \mu+v) \mathrm{t}}+\mathrm{dh}^{2} \\
& \left.\mathrm{e}^{-(\mu+2 v) \mathrm{t}}\right\}+3 \mathrm{t}^{2}\left\{\mathrm{ab}^{2} \mathrm{e}^{-3 \lambda \mathrm{t}}+\mathrm{b}^{2} \mathrm{ce}^{-(2 \lambda+\mu) \mathrm{t}}+2 \mathrm{abd}\right. \\
& \mathrm{e}^{-(2 \lambda+\mu) \mathrm{t}}+\mathrm{b}^{2} h \mathrm{e}^{-(2 \lambda+v) \mathrm{t}}+\mathrm{ad}^{2} \mathrm{e}^{-(\lambda+2 \mu) \mathrm{t}}+2 \mathrm{bcd} \\
& \mathrm{e}^{-(\lambda+2 \mu) \mathrm{t}}+2 b d h \mathrm{e}^{-(\lambda+\mu+v) \mathrm{t}}+\mathrm{cd}^{2} \mathrm{e}^{-3 \mu \mathrm{t}}+\mathrm{d}^{2} \mathrm{~h} \\
& \left.\mathrm{e}^{-(2 \mu+v) t}\right\}+\mathrm{t}^{3}\left\{\mathrm{~b}^{3} \mathrm{e}^{-3 \lambda \mathrm{t}}+\mathrm{b}^{2} \mathrm{de}^{-(2 \lambda+\mu) \mathrm{t}}+\mathrm{bd}^{2}\right. \\
& \left.\mathrm{e}^{-(\lambda+2 \mu) t}+\mathrm{d}^{3} \mathrm{e}^{-3 \mu \mathrm{t}}\right\}
\end{aligned}
$$

Now comparing equations (6) and (14), we obtain

$$
\begin{aligned}
& \sum_{i, j, k, l=0}^{\infty} F_{0,1}(a, b, c, d, h) e^{-(i \lambda+j \mu+k v) t}=a^{3} e^{-3 \lambda t} \\
& +3 \mathrm{a}^{2} \mathrm{ce}^{-(2 \lambda+\mu) \mathrm{t}}+3 \mathrm{a}^{2} \mathrm{he} \mathrm{e}^{-(2 \lambda+v) \mathrm{t}}+3 \mathrm{ac}^{2} \\
& \mathrm{e}^{-(\lambda+2 \mu) \mathrm{t}}+6 \mathrm{ache}^{-(\lambda+\mu+v) \mathrm{t}}+3 \mathrm{ah}^{2} \mathrm{e}^{-(\lambda+2 v) \mathrm{t}} \\
& +\mathrm{c}^{3} \mathrm{e}^{-3 \mu \mathrm{t}}+3 \mathrm{c}^{2} \mathrm{~h} \mathrm{e}^{-(2 \mu+v) \mathrm{t}}+3 \mathrm{ch}^{2} \mathrm{e}^{-(\mu+2 v) \mathrm{t}} \\
& +\mathrm{h}^{3} \mathrm{e}^{-3 \mathrm{vt}} \\
& \sum_{i, j, k, l=0}^{\infty} F_{1,1}(a, b, c, d, h) e^{-(i \lambda+j \mu+k v) t}=3\left\{a^{2} b e^{-3 \lambda t}\right. \\
& +2 a b c e^{-(2 \lambda+\mu) t}+a^{2} \mathrm{de}^{-(2 \lambda+\mu) t}+2 a b h e^{-(2 \lambda+v) t} \\
& +2 \mathrm{acde}^{-(\lambda+2 \mu) \mathrm{t}}+\mathrm{bc}^{2} \mathrm{e}^{-(\lambda+2 \mu) \mathrm{t}}+2 \mathrm{adhe}^{-(\lambda+\mu+v) \mathrm{t}} \\
& +2 b c h e^{-(\lambda+\mu+v) t}+b^{2} e^{-(\lambda+2 v) t}+c^{2} \mathrm{de}^{-3 \mu t} \\
& \left.+2 c d h e^{-(2 \mu+v) t}+{d h^{2}}^{-(\mu+2 v) t}\right\} \\
& \sum_{\mathrm{i}, \mathrm{j}, \mathrm{k}, \mathrm{l}=0}^{\infty} \mathrm{F}_{2,1}(\mathrm{a}, \mathrm{b}, \mathrm{c}, \mathrm{d}, \mathrm{h}) \mathrm{e}^{-(\mathrm{i} \lambda+\mathrm{j} \mu+\mathrm{kv}) \mathrm{t}}=3\left\{\mathrm{ab}^{2} \mathrm{e}^{-3 \lambda \mathrm{t}}\right. \\
& +\mathrm{b}^{2} \mathrm{ce}^{-(2 \lambda+\mu) \mathrm{t}}+2 \mathrm{abde}^{-(2 \lambda+\mu) \mathrm{t}}+\mathrm{b}^{2} \mathrm{he}^{-(2 \lambda+v) \mathrm{t}} \\
& +\mathrm{ad}^{2} \mathrm{e}^{-(\lambda+2 \mu) \mathrm{t}}+2 \mathrm{bcde} \mathrm{e}^{-(\lambda+2 \mu) \mathrm{t}}+2 \mathrm{bdh} \mathrm{e}^{-(\lambda+\mu+v) \mathrm{t}} \\
& \left.+c d^{2} \mathrm{e}^{-3 \mu \mathrm{t}}+\mathrm{d}^{2} h \mathrm{e}^{-(2 \mu+v) t}\right\} \\
& \sum_{\mathrm{i}, \mathrm{j}, \mathrm{k}, \mathrm{l}=0}^{\infty} \mathrm{F}_{3,1}(\mathrm{a}, \mathrm{b}, \mathrm{c}, \mathrm{d}, \mathrm{h}) \mathrm{e}^{-(\mathrm{i} \lambda+\mathrm{j} \mu+\mathrm{kv}) \mathrm{t}}=\mathrm{b}^{3} \mathrm{e}^{-3 \lambda t} \\
& +b^{2} d e^{-(2 \lambda+\mu) t}+b d^{2} e^{-(\lambda+2 \mu) t}+d^{3} e^{-3 \mu t}
\end{aligned}
$$

For equation (13), the equations (9) to (11) respectively become 


$$
\begin{aligned}
& (D+\lambda)^{2}(D+\mu)^{2}(D+v) u_{1}=-3 t^{2}\left\{a b^{2} e^{-3 \lambda t}\right. \\
& +\mathrm{b}^{2} \mathrm{ce}^{-(2 \lambda+\mu) \mathrm{t}}+2 \mathrm{abde} \mathrm{e}^{-(2 \lambda+\mu) \mathrm{t}}+\mathrm{b}^{2} \mathrm{he}^{-(2 \lambda+v) \mathrm{t}} \\
& +\operatorname{ad}^{2} \mathrm{e}^{-(\lambda+2 \mu) \mathrm{t}}+2 \mathrm{bcde}^{-(\lambda+2 \mu) \mathrm{t}}+2 \mathrm{bdhe}^{-(\lambda+\mu+v) t} \\
& \left.+c d^{2} e^{-3 \mu t}+d^{2} h e^{-(2 \mu+v) t}\right\}-t^{3}\left\{b^{3} e^{-3 \lambda t}\right. \\
& \left.+b^{2} d e^{-(2 \lambda+\mu) t}+b d^{2} e^{-(\lambda+2 \mu) t}+d^{3} e^{-3 \mu t}\right\} \\
& \mathrm{e}^{-\lambda \mathrm{t}}(\mathrm{D}+\mu-\lambda)^{2}(\mathrm{D}+\mathrm{v}-\lambda)\left(\frac{\partial \mathrm{A}_{1}}{\partial \mathrm{t}}+2 \mathrm{~B}_{1}\right) \\
& +\mathrm{e}^{-\mu \mathrm{t}}(\mathrm{D}+\lambda-\mu)^{2}(\mathrm{D}+\mathrm{v}-\mu)\left(\frac{\partial \mathrm{C}_{1}}{\partial \mathrm{t}}+2 \mathrm{D}_{1}\right) \\
& +\mathrm{e}^{-v t}(\mathrm{D}+\lambda-\mathrm{v})^{2}(\mathrm{D}+\mu-\mathrm{v})^{2} \mathrm{H}_{1}= \\
& -\left\{\mathrm{a}^{3} \mathrm{e}^{-3 \lambda t}+3 \mathrm{a}^{2} \mathrm{ce}^{-(2 \lambda+\mu) \mathrm{t}}\right. \\
& +3 \mathrm{a}^{2} h \mathrm{e}^{-(2 \lambda+v) \mathrm{t}}+3 \mathrm{ac}^{2} \mathrm{e}^{-(\lambda+2 \mu) \mathrm{t}} \\
& +6 \operatorname{ache}^{-(\lambda+\mu+v) t}+3 \mathrm{ah}^{2} \mathrm{e}^{-(\lambda+2 v) t} \\
& +c^{3} \mathrm{e}^{-3 \mu t}+3 \mathrm{c}^{2} h \mathrm{e}^{-(2 \mu+v) t} \\
& \left.+3 \operatorname{ch}^{2} \mathrm{e}^{-(\mu+2 v) \mathrm{t}}+\mathrm{h}^{3} \mathrm{e}^{-3 \mathrm{vt}}\right\} \\
& \mathrm{e}^{-\lambda \mathrm{t}}(\mathrm{D}+\mu-\lambda)^{2}(\mathrm{D}+\mathrm{v}-\lambda) \frac{\partial \mathrm{B}_{1}}{\partial \mathrm{t}} \\
& +\mathrm{e}^{-\mu \mathrm{t}}(\mathrm{D}+\lambda-\mu)^{2}(\mathrm{D}+\mathrm{v}-\mu) \frac{\partial \mathrm{D}_{1}}{\partial \mathrm{t}}= \\
& -3\left\{\mathrm{a}^{2} \mathrm{be}^{-3 \lambda \mathrm{t}}+2 \mathrm{abce} \mathrm{e}^{-(2 \lambda+\mu) \mathrm{t}}+\mathrm{a}^{2} \mathrm{de}^{-(2 \lambda+\mu) \mathrm{t}}\right. \\
& +2 a b h e^{-(2 \lambda+v) t}+2 \mathrm{acde}^{-(\lambda+2 \mu) \mathrm{t}}+\mathrm{bc}^{2} \mathrm{e}^{-(\lambda+2 \mu) \mathrm{t}} \\
& +2 a d h e^{-(\lambda+\mu+v) t}+2 b c h e^{-(\lambda+\mu+v) t}+b^{2} e^{-(\lambda+2 v) t} \\
& \left.+\mathrm{c}^{2} \mathrm{de}^{-3 \mu \mathrm{t}}+2 \mathrm{cdh} \mathrm{e}^{-(2 \mu+v) \mathrm{t}}+\mathrm{dh}^{2} \mathrm{e}^{-(\mu+2 v) \mathrm{t}}\right\}
\end{aligned}
$$

Since the relations $\lambda \gg>\mu$ and $\lambda<<v$ among the eigenvalues, then the equation (18) can be separated for the unknown functions $B_{1}$ and $D_{1}$ in the following way:

$$
\begin{aligned}
& \mathrm{e}^{-\lambda \mathrm{t}}(\mathrm{D}+\mu-\lambda)^{2}(\mathrm{D}+v-\lambda) \frac{\partial \mathrm{B}_{1}}{\partial \mathrm{t}}= \\
& -3\left\{\mathrm{a}^{2} \mathrm{be}^{-3 \lambda \mathrm{t}}+2 \mathrm{abce}^{-(2 \lambda+\mu) \mathrm{t}}+\mathrm{a}^{2} \mathrm{de}^{-(2 \lambda+\mu) \mathrm{t}}\right. \\
& +2 \mathrm{abhe}^{-(2 \lambda+v) \mathrm{t}}+2 \mathrm{acde}^{-(\lambda+2 \mu) \mathrm{t}}+\mathrm{bc}^{2} \mathrm{e}^{-(\lambda+2 \mu) \mathrm{t}} \\
& +2 \mathrm{adhe}^{-(\lambda+\mu+v) \mathrm{t}}+2 \mathrm{bch} \mathrm{e}^{-(\lambda+\mu+v) \mathrm{t}}+\mathrm{bh}^{2} \mathrm{e}^{-(\lambda+2 v) \mathrm{t}} \\
& \left.+2 \mathrm{cdhe}^{-(2 \mu+v) \mathrm{t}}+\mathrm{dh}^{2} \mathrm{e}^{-(\mu+2 v) \mathrm{t}}\right\} \\
& \mathrm{e}^{-\mu \mathrm{t}}(\mathrm{D}+\lambda-\mu)^{2}(\mathrm{D}+v-\mu) \frac{\partial \mathrm{D}_{1}}{\partial \mathrm{t}}=-3 \mathrm{c}^{2} \mathrm{de}^{-3 \mu \mathrm{t}}
\end{aligned}
$$

Solving equations (19) and (20), we get

$$
\begin{aligned}
\mathrm{B}_{1} & =1_{1} \mathrm{a}^{2} \mathrm{be}^{-2 \lambda \mathrm{t}}+1_{2} \mathrm{abce}^{-(\lambda+\mu) \mathrm{t}}+1_{3} \mathrm{a}^{2} \mathrm{de}^{-(\lambda+\mu) \mathrm{t}} \\
& +1_{4} \mathrm{abhe}^{-(\lambda+v) \mathrm{t}}+1_{5}\left(2 \mathrm{acd}+\mathrm{bc}^{2}\right) \mathrm{e}^{-2 \mu \mathrm{t}} \\
& +1_{6}(\mathrm{adh}+\mathrm{bch}) \mathrm{e}^{-(\mu+v) \mathrm{t}}+1_{7} \mathrm{cdhe}^{-(2 \mu+v-\lambda) \mathrm{t}} \\
& +1_{8} \mathrm{dh}^{2} \mathrm{e}^{-(\mu+2 v-\lambda) \mathrm{t}}+1_{9} \mathrm{bh}^{2} \mathrm{e}^{-(\lambda+2 v) \mathrm{t}}
\end{aligned}
$$

$$
\mathrm{D}_{1}=\mathrm{mc}^{2} \mathrm{de}^{-2 \mu \mathrm{t}}
$$

Where

$$
\begin{aligned}
& 1_{1}=\frac{3}{2 \lambda(3 \lambda-\mu)^{2}(v-3 \lambda)}, \quad 1_{2}=\frac{3}{2 \lambda^{2}(\lambda+\mu)(v-\mu-2 \lambda)}, \\
& 1_{3}=\frac{3}{4 \lambda^{2}(\lambda+\mu)(v-\mu-2 \lambda)}, \quad 1_{4}=\frac{-3}{\lambda(\lambda+v)(2 \lambda+v-\mu)^{2}}, \\
& 1_{5}=\frac{3}{2 \mu(\lambda+\mu)^{2}(v-\lambda-2 \mu)}, \quad 1_{6}=\frac{-6}{(\lambda+\mu)(\mu+v)(\lambda+v)^{2}}, \\
& 1_{7}=\frac{-3}{\mu(\mu+v)^{2}(2 \mu+v-\lambda)}, \quad 1_{8}=\frac{-3}{4 v^{2}(\mu+v)(\mu+2 v-\lambda)}, \\
& 1_{9}=\frac{-3}{2 v(\lambda+v)(\lambda+2 v-\mu)^{2}}, \quad m=\frac{3}{2 \mu(\lambda-3 \mu)^{2}(v-3 \mu)}
\end{aligned}
$$

Using the values of $B_{1}$ and $D_{1}$ in equation (17), we obtain

$$
\begin{aligned}
& \mathrm{e}^{-\lambda \mathrm{t}}(\mathrm{D}+\mu-\lambda)^{2}(\mathrm{D}+\mathrm{v}-\lambda) \frac{\partial \mathrm{A}_{1}}{\partial \mathrm{t}}+\mathrm{e}^{-\mu \mathrm{t}} \\
& (\mathrm{D}+\lambda-\mu)^{2}(\mathrm{D}+\mathrm{v}-\mu) \frac{\partial \mathrm{C}_{1}}{\partial \mathrm{t}}+\mathrm{e}^{-\mathrm{vt}} \\
& (D+\lambda-v)^{2}(D+\mu-v)^{2} H_{1}=-a^{3} e^{-3 \lambda t} \\
& -3 \mathrm{a}^{2} \mathrm{ce}^{-(2 \lambda+\mu) \mathrm{t}}-3 \mathrm{a}^{2} h \mathrm{e}^{-(2 \lambda+v) \mathrm{t}}-3 \mathrm{ac}^{2} \\
& \mathrm{e}^{-(\lambda+2 \mu) \mathrm{t}}-6 \mathrm{ache}^{-(\lambda+\mu+v) \mathrm{t}}-3 \mathrm{ah}^{2} \mathrm{e}^{-(\lambda+2 v) \mathrm{t}} \\
& -c^{3} e^{-3 \mu t}-3 c^{2} h e^{-(2 \mu+v) t}-3 \operatorname{ch}^{2} e^{-(\mu+2 v) t} \\
& -\mathrm{h}^{3} \mathrm{e}^{-3 v \mathrm{t}}-2(3 \lambda-\mu)^{2}(v-3 \lambda) \mathrm{l}_{1} \mathrm{a}^{2} b \mathrm{e}^{-3 \lambda t} \\
& -8 \lambda^{2}(v-\mu-2 \lambda) l_{2} \mathrm{abce}^{-(2 \lambda+\mu) \mathrm{t}}-8 \lambda^{2} \\
& (v-\mu-2 \lambda) 1_{3} \mathrm{a}^{2} \mathrm{de}^{-(2 \lambda+\mu) \mathrm{t}}+4 \lambda(\mu-v-2 \lambda)^{2} \\
& 1_{4} \mathrm{abhe}^{-(2 \lambda+v) \mathrm{t}}-2(\lambda+\mu)^{2}(v-2 \mu-\lambda) 1_{5} \\
& \left(2 \mathrm{acd}+\mathrm{bc}^{2}\right) \mathrm{e}^{-(\lambda+2 \mu) \mathrm{t}}+2(\lambda+v)^{2}(\lambda+\mu) \\
& 1_{6}(\mathrm{adh}+\mathrm{bch}) \mathrm{e}^{-(\lambda+\mu+v) \mathrm{t}}+4 \mu(\mu+v) 1_{7} \mathrm{cdh} \\
& \mathrm{e}^{-(2 \mu+v) \mathrm{t}}+8 \mathrm{v}^{2}(\mu+v) 1_{8} \mathrm{dh}^{2} \mathrm{e}^{-(\mu+2 v) \mathrm{t}} \\
& +2(\lambda+2 v-\mu)^{2}(\lambda+v) l_{9} b^{2} \mathrm{e}^{-(\lambda+2 v) \mathrm{t}} \\
& -2(\lambda-3 \mu)^{2}(v-3 \mu) \mathrm{mc}^{2} \mathrm{de}^{-3 \mu \mathrm{t}}
\end{aligned}
$$

Again, applying the conditions $\lambda>>\mu$ and $\lambda<<v$ in equation (23), we obtain the following equations for unknown functions $\mathrm{A}_{1}, \mathrm{C}_{1}$ and $\mathrm{H}_{1}$ :

$$
\begin{aligned}
\mathrm{e}^{-\lambda \mathrm{t}}(\mathrm{D}+ & \mu-\lambda)^{2}(\mathrm{D}+\mathrm{v}-\lambda) \frac{\partial \mathrm{A}_{1}}{\partial \mathrm{t}}=-\mathrm{a}^{3} \mathrm{e}^{-3 \lambda \mathrm{t}} \\
& -3 \mathrm{a}^{2} \mathrm{ce}^{-(2 \lambda+\mu) \mathrm{t}}-3 \mathrm{ac}^{2} \mathrm{e}^{-(\lambda+2 \mu) \mathrm{t}} \\
& -2(3 \lambda-\mu)^{2}(\nu-3 \lambda) \mathrm{l}_{1} \mathrm{a}^{2} \mathrm{be}^{-3 \lambda \mathrm{t}} \\
& -8 \lambda^{2}(\nu-\mu-2 \lambda) 1_{2} \mathrm{abce}^{-(2 \lambda+\mu) \mathrm{t}} \\
- & 8 \lambda^{2}(v-\mu-2 \lambda) 1_{3} \mathrm{a}^{2} \mathrm{de}^{-(2 \lambda+\mu) \mathrm{t}} \\
& -2(\lambda+\mu)^{2}(v-2 \mu-\lambda) 1_{5} \\
& \left(2 \mathrm{acd}+\mathrm{bc}^{2}\right) \mathrm{e}^{-(\lambda+2 \mu) \mathrm{t}}
\end{aligned}
$$




$$
\begin{array}{r}
\mathrm{e}^{-\mu \mathrm{t}}(\mathrm{D}+\lambda-\mu)^{2}(\mathrm{D}+\nu-\mu) \frac{\partial \mathrm{C}_{1}}{\partial \mathrm{t}}=-\mathrm{c}^{3} \mathrm{e}^{-3 \mu \mathrm{t}} \\
-2(\lambda-3 \mu)^{2}(\nu-3 \mu) \mathrm{mc}^{2} \mathrm{de}^{-3 \mu \mathrm{t}}
\end{array}
$$

$\mathrm{e}^{-v \mathrm{t}}(\mathrm{D}+\lambda-v)^{2}(\mathrm{D}+\mu-v)^{2} \mathrm{H}_{1}=-3 \mathrm{a}^{2} \mathrm{he}^{-(2 \lambda+v) \mathrm{t}}$

$-6 a c h e^{-(\lambda+\mu+v) t}-3 a^{2} \mathrm{e}^{-(\lambda+2 v) t}-3 \mathrm{c}^{2} h \mathrm{e}^{-(2 \mu+v) t}$

$-3 \mathrm{ch}^{2} \mathrm{e}^{-(\mu+2 v) t}-\mathrm{h}^{3} \mathrm{e}^{-3 v \mathrm{t}}+4 \lambda(\mu-v-2 \lambda)^{2} 1_{4} \mathrm{abh}$

$\mathrm{e}^{-(2 \lambda+v) \mathrm{t}}+2(\lambda+v)^{2}(\lambda+\mu) 1_{6}(\mathrm{adh}+\mathrm{bch}) \mathrm{e}^{-(\lambda+\mu+v) \mathrm{t}}$

$+4 \mu(\mu+v) 1_{7} \mathrm{cdh}^{-(2 \mu+v) t}+8 v^{2}(\mu+v) 1_{8} \mathrm{dh}^{2} \mathrm{e}^{-(\mu+2 v) \mathrm{t}}$

$+2(\lambda+2 v-\mu)^{2}(\lambda+v) 1_{9} b h^{2} e^{-(\lambda+2 v) t}$

Solving equations (24), (25) and (26), we obtain

$$
\begin{aligned}
\mathrm{A}_{1}= & \mathrm{r}_{1} \mathrm{a}^{3} \mathrm{e}^{-2 \lambda t}+\mathrm{r}_{2} \mathrm{a}^{2} \mathrm{ce}^{-(\lambda+\mu) \mathrm{t}}+\mathrm{r}_{3} \mathrm{ac}^{2} \mathrm{e}^{-2 \mu t} \\
+ & \mathrm{r}_{4} \mathrm{a}^{2} \mathrm{be}^{-2 \lambda t}+\mathrm{r}_{5} a b c e^{-(\lambda+\mu) t}+\mathrm{r}_{6} \mathrm{a}^{2} \mathrm{~d} \\
\mathrm{e}^{-(\lambda+\mu) t}+\mathrm{r}_{7}\left(2 \mathrm{acd}+\mathrm{bc}^{2}\right) \mathrm{e}^{-2 \mu \mathrm{t}} & \\
& \mathrm{C}_{1}=\mathrm{n}_{1} \mathrm{c}^{3} \mathrm{e}^{-2 \mu \mathrm{t}}+\mathrm{n}_{2} \mathrm{c}^{2} \mathrm{de}^{-2 \mu t}
\end{aligned}
$$

$\mathrm{H}_{1}=\mathrm{s}_{1} \mathrm{a}^{2} \mathrm{he}^{-2 \lambda t}+\mathrm{s}_{2} \mathrm{ache}^{-(\lambda+\mu) \mathrm{t}}+\mathrm{s}_{3} \mathrm{ah}^{2} \mathrm{e}^{-(\lambda+v) \mathrm{t}}$

$$
+\mathrm{s}_{4} \mathrm{c}^{2} \mathrm{he}^{-2 \mu \mathrm{t}}+\mathrm{s}_{5} \mathrm{ch}^{2} \mathrm{e}^{-(\mu+v) \mathrm{t}}+\mathrm{s}_{6} \mathrm{~h}^{3} \mathrm{e}^{-2 v \mathrm{t}}
$$$$
+\mathrm{s}_{7} \mathrm{abhe}^{-2 \lambda t}+\mathrm{s}_{8}(\mathrm{adh}+\mathrm{bch}) \mathrm{e}^{-(\lambda+\mu) t}
$$$$
+\mathrm{s}_{9} \mathrm{cdhe}^{-2 \mu \mathrm{t}}+\mathrm{s}_{10} \mathrm{dh}^{2} \mathrm{e}^{-(\mu+v) \mathrm{t}}+\mathrm{s}_{11} \mathrm{bh}^{2} \mathrm{e}^{-(\lambda+v) \mathrm{t}}
$$

The solution of the equation (3.3.4) for $\mathrm{u}_{1}$ is

$$
\begin{aligned}
& \mathrm{u}_{1}=a b^{2} \mathrm{e}^{-3 \lambda \mathrm{t}}\left(\mathrm{w}_{1} \mathrm{t}^{2}+\mathrm{w}_{2} \mathrm{t}+\mathrm{w}_{3}\right)+\left(\mathrm{b}^{2} \mathrm{c}+2 \mathrm{abd}\right) \\
& \mathrm{e}^{-(2 \lambda+\mu) t}\left(\mathrm{w}_{4} \mathrm{t}^{2}+\mathrm{w}_{5} \mathrm{t}+\mathrm{w}_{6}\right)+\mathrm{b}^{2} h \mathrm{e}^{-(2 \lambda+v) t} \\
&\left(\mathrm{w}_{7} \mathrm{t}^{2}+\mathrm{w}_{8} \mathrm{t}+\mathrm{w}_{9}\right)+\left(\mathrm{ad}^{2}+2 \mathrm{bcd}\right) \mathrm{e}^{-(\lambda+2 \mu) \mathrm{t}} \\
&\left(\mathrm{w}_{10} \mathrm{t}^{2}+\mathrm{w}_{11} \mathrm{t}+\mathrm{w}_{12}\right)+\mathrm{bdhe} \mathrm{e}^{-(\lambda+\mu+v) \mathrm{t}}\left(\mathrm{w}_{13} \mathrm{t}^{2}\right. \\
&\left.+\mathrm{w}_{14} \mathrm{t}+\mathrm{w}_{15}\right)+\mathrm{cd}^{2} \mathrm{e}^{-3 \mu \mathrm{t}}\left(\mathrm{w}_{16} \mathrm{t}^{2}+\mathrm{w}_{17} \mathrm{t}+\mathrm{w}_{18}\right) \\
&+\mathrm{d}^{2} \mathrm{he}^{-(2 \mu+v) \mathrm{t}}\left(\mathrm{w}_{19} \mathrm{t}^{2}+\mathrm{w}_{20} \mathrm{t}+\mathrm{w}_{21}\right)+\mathrm{b}^{3} \mathrm{e}^{-3 \lambda t} \\
&\left(\mathrm{w}_{22} \mathrm{t}^{3}+\mathrm{w}_{23} \mathrm{t}^{2}+\mathrm{w}_{24} \mathrm{t}+\mathrm{w}_{25}\right)+\mathrm{b}^{2} \mathrm{de}^{-(2 \lambda+\mu) \mathrm{t}} \\
&\left(\mathrm{w}_{26} \mathrm{t}^{3}+\mathrm{w}_{27} \mathrm{t}^{2}+\mathrm{w}_{28} \mathrm{t}+\mathrm{w}_{29}\right)+\mathrm{bd}^{2} \mathrm{e}^{-(\lambda+2 \mu) \mathrm{t}} \\
&\left(\mathrm{w}_{30} \mathrm{t}^{3}+\mathrm{w}_{31} \mathrm{t}^{2}+\mathrm{w}_{32} \mathrm{t}+\mathrm{w}_{33}\right)+\mathrm{d}^{3} \mathrm{e}^{-3 \mu \mathrm{t}} \\
&\left(\mathrm{w}_{34} \mathrm{t}^{3}+\mathrm{w}_{35} \mathrm{t}^{2}+\mathrm{w}_{36} \mathrm{t}+\mathrm{w}_{37}\right)
\end{aligned}
$$

where $\mathrm{w}_{1}=\frac{1}{4 \lambda^{2}(3 \lambda-\mu)^{2}(3 \lambda-v)}$,

$$
\begin{gathered}
\mathrm{w}_{2}=\frac{2}{4 \lambda^{2}(3 \lambda-\mu)^{2}(3 \lambda-v)}\left(\frac{1}{\lambda}+\frac{2}{3 \lambda-\mu}+\frac{1}{3 \lambda-v}\right), \\
\mathrm{w}_{3}=\frac{2}{4 \lambda^{2}(3 \lambda-\mu)^{2}(3 \lambda-v)}\left\{\frac{3}{4 \lambda^{2}}+\frac{2}{\lambda(3 \lambda-\mu)}+\frac{3}{(3 \lambda-\mu)^{2}}\right. \\
\left.+\frac{1}{\lambda(3 \lambda-v)}+\frac{1}{(3 \lambda-v)^{2}}+\frac{2}{(3 \lambda-\mu)(3 \lambda-v)}\right\}, \\
\mathrm{w}_{4}=\frac{3}{4 \lambda^{2}(\lambda+\mu)^{2}(2 \lambda+\mu-v)},
\end{gathered}
$$

$$
\begin{aligned}
& \mathrm{w}_{5}=\frac{6}{4 \lambda^{2}(\lambda+\mu)^{2}(2 \lambda+\mu-v)}\left(\frac{2}{\lambda+\mu}+\frac{1}{\lambda}+\frac{1}{2 \lambda+\mu-v}\right), \\
& \mathrm{w}_{6}=\frac{3}{2 \lambda^{2}(\lambda+\mu)^{2}(2 \lambda+\mu-v)}\left\{\frac{3}{(\lambda+\mu)^{2}}+\frac{3}{4 \lambda^{2}}+\frac{2}{\lambda(\lambda+\mu)}\right. \\
& \left.+\frac{1}{(2 \lambda+\mu-v)^{2}}+\frac{1}{\lambda(2 \lambda+\mu-v)}+\frac{2}{(\lambda+\mu)(2 \lambda+\mu-v)}\right\} \text {, } \\
& \mathrm{w}_{7}=\frac{3}{2 \lambda(2 \lambda+v-\mu)^{2}(\lambda+v)^{2}}, \\
& \mathrm{w}_{8}=\frac{3}{\lambda(2 \lambda+v-\mu)^{2}(\lambda+v)^{2}}\left(\frac{2}{\lambda+v}+\frac{2}{2 \lambda+v-\mu}+\frac{1}{2 \lambda}\right) \text {, } \\
& \mathrm{w}_{9}=\frac{3}{\lambda(2 \lambda+v-\mu)^{2}(\lambda+v)^{2}}\left\{\frac{3}{(\lambda+v)^{2}}+\frac{3}{(2 \lambda+v-\mu)^{2}}\right. \\
& \left.+\frac{4}{(\lambda+v)(2 \lambda+v-\mu)}+\frac{1}{4 \lambda^{2}}+\frac{1}{\lambda(\lambda+v)}+\frac{1}{\lambda(2 \lambda+v-\mu)}\right\}, \\
& \mathrm{w}_{10}=\frac{3}{4 \mu^{2}(\lambda+\mu)^{2}(\lambda+2 \mu-v)}, \\
& \mathrm{w}_{11}=\frac{3}{2 \mu^{2}(\lambda+\mu)^{2}(\lambda+2 \mu-v)}\left(\frac{2}{\lambda+\mu}+\frac{1}{\mu}+\frac{1}{\lambda+2 \mu-v}\right), \\
& \mathrm{w}_{12}=\frac{3}{2 \mu^{2}(\lambda+\mu)^{2}(\lambda+2 \mu-v)} \\
& \left\{\frac{3}{(\lambda+\mu)^{2}}+\frac{3}{4 \mu^{2}}+\frac{2}{\mu(\lambda+\mu)}+\frac{1}{(\lambda+2 \mu-v)^{2}}\right. \\
& \left.+\frac{1}{\mu(\lambda+2 \mu-v)}+\frac{2}{(\lambda+\mu)(\lambda+2 \mu-v)}\right\}, \\
& \mathrm{w}_{13}=\frac{6}{(\lambda+\mu)(\mu+v)^{2}(\lambda+v)^{2}}, \\
& \mathrm{w}_{14}=\frac{12}{(\lambda+\mu)(\mu+v)^{2}(\lambda+v)^{2}}\left(\frac{1}{\lambda+\mu}+\frac{2}{\mu+v}+\frac{2}{\lambda+v}\right) \text {, } \\
& \mathrm{w}_{15}=\frac{12}{(\lambda+\mu)(\mu+v)^{2}(\lambda+v)^{2}} \\
& \left\{\frac{3}{(\lambda+v)^{2}}+\frac{3}{(\mu+v)^{2}}+\frac{4}{(\mu+v)(\lambda+v)}\right. \\
& \left.\frac{1}{(\lambda+\mu)^{2}}+\frac{2}{(\lambda+\mu)(\mu+v)}+\frac{2}{(\lambda+\mu)(\lambda+v)}\right\}, \\
& \mathrm{w}_{16}=\frac{3}{4 \mu^{2}(\lambda-3 \mu)^{2}(3 \mu-v)}, \\
& \mathrm{w}_{17}=\frac{3}{2 \mu^{2}(\lambda-3 \mu)^{2}(3 \mu-v)}\left(\frac{1}{\mu}+\frac{2}{3 \mu-\lambda}+\frac{1}{3 \mu-v}\right) \text {, } \\
& \mathrm{w}_{18}=\frac{3}{2 \mu^{2}(\lambda-3 \mu)^{2}(3 \mu-v)} \\
& \left\{\frac{3}{4 \mu^{2}}+\frac{2}{\mu(3 \mu-\lambda)}+\frac{3}{(3 \mu-\lambda)^{2}}+\frac{1}{\mu(3 \mu-v)}\right. \\
& \left.+\frac{1}{(3 \mu-v)^{2}}+\frac{2}{(3 \mu-\lambda)(3 \mu-v)}\right\} \text {, }
\end{aligned}
$$




$$
\begin{aligned}
& \mathrm{w}_{19}=\frac{3}{2 \mu(2 \mu+v-\lambda)^{2}(\mu+v)^{2}}, \\
& \mathrm{w}_{20}=\frac{3}{\mu(2 \mu+v-\lambda)^{2}(\mu+v)^{2}}\left(\frac{2}{\mu+v}+\frac{2}{2 \mu+v-\lambda}+\frac{1}{2 \mu}\right) \text {, } \\
& \mathrm{w}_{21}=\frac{3}{\mu(2 \mu+v-\lambda)^{2}(\mu+v)^{2}}\left\{\frac{3}{(\mu+v)^{2}}+\frac{3}{(2 \mu+v-\lambda)^{2}}\right. \\
& \left.+\frac{4}{(\mu+v)(2 \mu+v-\lambda)}+\frac{1}{4 \mu^{2}}+\frac{1}{\mu(\mu+v)}+\frac{1}{\mu(2 \mu+v-\lambda)}\right\}, \\
& \mathrm{w}_{22}=\frac{1}{4 \lambda^{2}(3 \lambda-\mu)^{2}(3 \lambda-v)}, \\
& \mathrm{w}_{23}=\frac{3}{4 \lambda^{2}(3 \lambda-\mu)^{2}(3 \lambda-v)}\left(\frac{1}{\lambda}+\frac{2}{3 \lambda-\mu}+\frac{1}{3 \lambda-v}\right), \\
& \mathrm{w}_{24}=\frac{3}{2 \lambda^{2}(3 \lambda-\mu)^{2}(3 \lambda-v)} \\
& \left\{\frac{3}{4 \lambda^{2}}+\frac{2}{\lambda(3 \lambda-\mu)}+\frac{3}{(3 \lambda-\mu)^{2}}+\frac{1}{\lambda(3 \lambda-v)}\right. \\
& \left.+\frac{1}{(3 \lambda-v)^{2}}+\frac{2}{(3 \lambda-\mu)(3 \lambda-v)}\right\} \text {, } \\
& \mathrm{w}_{25}=\frac{3}{2 \lambda^{2}(3 \lambda-\mu)^{2}(3 \lambda-v)}\left\{\frac{1}{2 \lambda^{3}}\right. \\
& +\frac{3}{2 \lambda^{2}(3 \lambda-\mu)}+\frac{3}{\lambda(3 \lambda-\mu)^{2}}+\frac{4}{(3 \lambda-\mu)^{3}} \\
& +\frac{1}{\lambda(3 \lambda-v)^{2}}+\frac{2}{(3 \lambda-\mu)(3 \lambda-v)^{2}}+\frac{1}{(3 \lambda-v)^{3}} \\
& \left.+\frac{3}{4 \lambda^{2}(3 \lambda-v)}+\frac{2}{\lambda(3 \lambda-\mu)(3 \lambda-v)}+\frac{3}{(3 \lambda-\mu)^{2}(3 \lambda-v)}\right\}, \\
& \mathrm{w}_{26}=\frac{3}{4 \lambda^{2}(\lambda+\mu)^{2}(2 \lambda+\mu-v)}, \\
& \mathrm{w}_{27}=\frac{9}{4 \lambda^{2}(\lambda+\mu)^{2}(2 \lambda+\mu-v)}\left(\frac{2}{\lambda+\mu}+\frac{1}{\lambda}+\frac{1}{2 \lambda+\mu-v}\right), \\
& \mathrm{w}_{28}=\frac{9}{2 \lambda^{2}(\lambda+\mu)^{2}(2 \lambda+\mu-v)} \\
& \left\{\frac{3}{(\lambda+\mu)^{2}}+\frac{3}{4 \lambda^{2}}+\frac{2}{\lambda(\lambda+\mu)}+\frac{1}{(2 \lambda+\mu-v)^{2}}\right. \\
& \left.+\frac{1}{\lambda(2 \lambda+\mu-v)}+\frac{2}{(\lambda+\mu)(2 \lambda+\mu-v)}\right\} \text {, } \\
& \mathrm{w}_{29}=\frac{9}{2 \lambda^{2}(\lambda+\mu)^{2}(2 \lambda+\mu-v)}\left\{\frac{4}{(\lambda+\mu)^{3}}+\frac{3}{2 \lambda^{3}}\right. \\
& +\frac{3}{\lambda(\lambda+\mu)^{2}}+\frac{3}{2 \lambda^{2}(\lambda+\mu)}+\frac{1}{(2 \lambda+\mu-v)^{3}}+\frac{2}{(\lambda+\mu)(2 \lambda+\mu-v)^{2}} \\
& +\frac{1}{\lambda(2 \lambda+\mu-v)^{2}}+\frac{2}{(\lambda+\mu)^{2}(2 \lambda+\mu-v)} \\
& \left.+\frac{3}{4 \lambda^{2}(2 \lambda+\mu-v)}+\frac{2}{\lambda(\lambda+\mu)(2 \lambda+\mu-v)}\right\} \text {, }
\end{aligned}
$$

$$
\begin{aligned}
& \mathrm{w}_{30}=\frac{3}{4 \mu^{2}(\lambda+\mu)^{2}(\lambda+2 \mu-v)}, \\
& \mathrm{w}_{31}=\frac{9}{4 \mu^{2}(\lambda+\mu)^{2}(\lambda+2 \mu-v)}\left(\frac{2}{\lambda+\mu}+\frac{1}{\mu}+\frac{1}{\lambda+2 \mu-v}\right) \text {, } \\
& \mathrm{w}_{32}=\frac{9}{2 \mu^{2}(\lambda+\mu)^{2}(\lambda+2 \mu-v)}\left\{\frac{3}{(\lambda+\mu)^{2}}+\frac{3}{4 \mu^{2}}+\frac{2}{\mu(\lambda+\mu)}\right. \\
& \left.+\frac{1}{(\lambda+2 \mu-v)^{2}}+\frac{1}{\mu(\lambda+2 \mu-v)}+\frac{2}{(\lambda+\mu)(\lambda+2 \mu-v)}\right\}, \\
& \mathrm{w}_{33}=\frac{9}{2 \mu^{2}(\lambda+\mu)^{2}(\lambda+2 \mu-v)}\left\{\frac{4}{(\lambda+\mu)^{3}}\right. \\
& +\frac{3}{2 \mu^{2}(\lambda+\mu)}+\frac{1}{(\lambda+2 \mu-v)^{3}}+\frac{3}{2 \mu^{3}} \\
& +\frac{3}{\mu(\lambda+\mu)^{2}}+\frac{2}{(\lambda+\mu)(\lambda+2 \mu-v)^{2}} \\
& +\frac{1}{\mu(\lambda+2 \mu-v)^{2}}+\frac{3}{(\lambda+\mu)^{2}(\lambda+2 \mu-v)} \\
& \left.+\frac{3}{4 \mu^{2}(\lambda+2 \mu-v)}+\frac{2}{\mu(\lambda+\mu)(\lambda+2 \mu-v)}\right\}, \\
& \mathrm{w}_{34}=\frac{1}{4 \mu^{2}(\lambda-3 \mu)^{2}(3 \mu-v)}, \\
& \mathrm{w}_{35}=\frac{3}{4 \mu^{2}(\lambda-3 \mu)^{2}(3 \mu-v)} \\
& \left(\frac{1}{\mu}+\frac{2}{3 \mu-\lambda}+\frac{1}{3 \mu-v}\right) \\
& \mathrm{w}_{36}=\frac{3}{2 \mu^{2}(\lambda-3 \mu)^{2}(3 \mu-v)}\left\{\frac{3}{4 \mu^{2}}\right. \\
& +\frac{2}{\mu(3 \mu-\lambda)}+\frac{3}{(3 \mu-\lambda)^{2}}+\frac{1}{\mu(3 \mu-v)} \\
& \left.+\frac{1}{(3 \mu-v)^{2}}+\frac{2}{(3 \mu-\lambda)(3 \mu-v)}\right\} \text {, } \\
& \mathrm{w}_{37}=\frac{3}{2 \mu^{2}(\lambda-3 \mu)^{2}(3 \mu-v)}\left\{\frac{1}{2 \mu^{3}}\right. \\
& +\frac{3}{2 \mu^{2}(3 \mu-\lambda)}+\frac{3}{\mu(3 \mu-\lambda)^{2}}+\frac{4}{(3 \mu-\lambda)^{3}} \\
& +\frac{1}{\mu(3 \mu-v)^{2}}+\frac{2}{(3 \mu-\lambda)(3 \mu-v)^{2}} \\
& +\frac{1}{(3 \mu-v)^{3}}+\frac{3}{4 \mu^{2}(3 \mu-v)} \\
& \left.+\frac{2}{\mu(3 \mu-\lambda)(3 \mu-v)}+\frac{3}{(3 \mu-\lambda)^{2}(3 \mu-v)}\right\}
\end{aligned}
$$

Substituting the values of $\mathrm{A}_{1}, \mathrm{~B}_{1}, \mathrm{C}_{1}, \mathrm{D}_{1}$ and $\mathrm{H}_{1}$ from equations (27), (21), (28), (22) and (29) into equation (4), we obtain 


$$
\begin{aligned}
& \dot{\mathrm{a}}=\varepsilon\left\{\mathrm{r}_{1} \mathrm{a}^{3} \mathrm{e}^{-2 \lambda \mathrm{t}}+\mathrm{r}_{2} \mathrm{a}^{2} \mathrm{ce}^{-(\lambda+\mu) \mathrm{t}}+\mathrm{r}_{3} \mathrm{ac}^{2} \mathrm{e}^{-2 \mu \mathrm{t}}\right. \\
& +\mathrm{r}_{4} \mathrm{a}^{2} b \mathrm{e}^{-2 \lambda \mathrm{t}}+\mathrm{r}_{5} \mathrm{abce} \mathrm{e}^{-(\lambda+\mu) \mathrm{t}}+\mathrm{r}_{6} \mathrm{a}^{2} d \mathrm{e}^{-(\lambda+\mu) \mathrm{t}} \\
& \left.+r_{7}\left(2 a c d+b c^{2}\right) e^{-2 \mu t}\right\} \\
& \dot{\mathrm{b}}=\varepsilon\left\{\mathrm{l}_{1} \mathrm{a}^{2} \mathrm{be} \mathrm{e}^{-2 \lambda \mathrm{t}}+\mathrm{l}_{2} \mathrm{abce} \mathrm{e}^{-(\lambda+\mu) \mathrm{t}}+\mathrm{l}_{3} \mathrm{a}^{2} \mathrm{de}^{-(\lambda+\mu) \mathrm{t}}\right. \\
& +1_{4} a b h e^{-(\lambda+v) t}+1_{5}\left(2 a c d+b^{2}\right) e^{-2 \mu t} \\
& +1_{6}(\operatorname{adh}+\mathrm{bch}) \mathrm{e}^{-(\mu+v) \mathrm{t}}+1_{7} \operatorname{cdhe}^{-(2 \mu+v-\lambda) \mathrm{t}} \\
& \left.+1_{8} \mathrm{dh}^{2} \mathrm{e}^{-(\mu+2 v-\lambda) \mathrm{t}}+1_{9} \mathrm{bh}^{2} \mathrm{e}^{-(\lambda+2 v) t}\right\} \\
& \dot{\mathrm{c}}=\varepsilon\left\{\mathrm{n}_{1} \mathrm{c}^{3} \mathrm{e}^{-2 \mu \mathrm{t}}+\mathrm{n}_{2} \mathrm{c}^{2} \mathrm{de}^{-2 \mu \mathrm{t}}\right\} \\
& \dot{\mathrm{d}}=\varepsilon \mathrm{mc}^{2} \mathrm{de}^{-2 \mu \mathrm{t}} \\
& \dot{\mathrm{h}}=\varepsilon\left\{\mathrm{s}_{1} \mathrm{a}^{2} \mathrm{he} \mathrm{e}^{-2 \lambda \mathrm{t}}+\mathrm{s}_{2} \mathrm{ache} \mathrm{e}^{-(\lambda+\mu) \mathrm{t}}\right. \\
& +\mathrm{s}_{3} \mathrm{ah}^{2} \mathrm{e}^{-(\lambda+v) \mathrm{t}}+\mathrm{s}_{4} \mathrm{c}^{2} h \mathrm{e}^{-2 \mu \mathrm{t}}+\mathrm{s}_{5} \mathrm{ch}^{2} \\
& \mathrm{e}^{-(\mu+v) \mathrm{t}}+\mathrm{s}_{6} \mathrm{~h}^{3} \mathrm{e}^{-2 v \mathrm{t}}+\mathrm{s}_{7} a b h \mathrm{e}^{-2 \lambda \mathrm{t}} \\
& +\mathrm{s}_{8}(\mathrm{adh}+\mathrm{bch}) \mathrm{e}^{-(\lambda+\mu) \mathrm{t}}+\mathrm{s}_{9} \text { cdhe } \mathrm{e}^{-2 \mu \mathrm{t}} \\
& \left.+\mathrm{s}_{10} \mathrm{dh}^{2} \mathrm{e}^{-(\mu+v) \mathrm{t}}+\mathrm{s}_{11} \mathrm{bh}^{2} \mathrm{e}^{-(\lambda+v) t}\right\}
\end{aligned}
$$

Here, all of the equations (31) have no exact solutions. However, since $\dot{a}, \dot{b}, \dot{c}, \dot{d}$ and $\dot{h}$ are proportional to the small parameter $\varepsilon$, they are slowly varying functions of time $t$. Consequently, it is possible to replace $a, b, c, d$ and $h$ by their respective values obtained in linear case (i.e., the values of $a$, $b, c, d$ and $h$ obtained when $\varepsilon=0$ ) in the right hand side of equations (31). This type of replacement was first introduced by Murty and Deekshatulu [24], and Mutry et.al. [19] to solve similar types of nonlinear equations. Therefore, the solutions of equation (31) are

$$
\begin{aligned}
\mathrm{a}= & \mathrm{a}_{0}+\varepsilon\left\{\mathrm{r}_{1} \mathrm{a}_{0}^{3} \frac{1-\mathrm{e}^{-2 \lambda \mathrm{t}}}{2 \lambda}+\mathrm{r}_{2} \mathrm{a}_{0}^{2} \mathrm{c}_{0} \frac{1-\mathrm{e}^{-(\lambda+\mu) \mathrm{t}}}{\lambda+\mu}\right. \\
+ & \mathrm{r}_{3} \mathrm{a}_{0} \mathrm{c}_{0}^{2} \frac{1-\mathrm{e}^{-2 \mu \mathrm{t}}}{2 \mu}+\mathrm{r}_{4} \mathrm{a}_{0}^{2} \mathrm{~b}_{0} \frac{1-\mathrm{e}^{-2 \lambda t}}{2 \lambda}+\mathrm{r}_{5} \mathrm{a}_{0} \mathrm{~b}_{0} \mathrm{c}_{0} \frac{1-\mathrm{e}^{-(\lambda+\mu) \mathrm{t}}}{\lambda+\mu} \\
+ & \left.\mathrm{r}_{6} \mathrm{a}_{0}^{2} \mathrm{~d}_{0} \frac{1-\mathrm{e}^{-(\lambda+\mu) \mathrm{t}}}{\lambda+\mu}+\mathrm{r}_{7}\left(2 \mathrm{a}_{0} \mathrm{c}_{0} \mathrm{~d}_{0}+\mathrm{b}_{0} \mathrm{c}_{0}^{2}\right) \frac{1-\mathrm{e}^{-2 \mu \mathrm{t}}}{2 \mu}\right\} \\
\mathrm{b}= & \mathrm{b}_{0}+\varepsilon\left\{\mathrm{l}_{1} \mathrm{a}_{0}^{2} \mathrm{~b}_{0} \frac{1-\mathrm{e}^{-2 \lambda \mathrm{t}}}{2 \lambda}+\mathrm{l}_{2} \mathrm{a}_{0} \mathrm{~b}_{0} \mathrm{c}_{0} \frac{1-\mathrm{e}^{-(\lambda+\mu) \mathrm{t}}}{\lambda+\mu}\right. \\
& +\mathrm{l}_{3} \mathrm{a}_{0}^{2} \mathrm{~d}_{0} \frac{1-\mathrm{e}^{-(\lambda+\mu) \mathrm{t}}}{\lambda+\mu}+\mathrm{l}_{4} \mathrm{a}_{0} \mathrm{~b}_{0} \mathrm{~h}_{0} \frac{1-\mathrm{e}^{-(\lambda+v) \mathrm{t}}}{\lambda+\mu} \\
& +\mathrm{l}_{5}\left(2 \mathrm{a}_{0} \mathrm{c}_{0} \mathrm{~d}_{0}+\mathrm{b}_{0} \mathrm{c}_{0}^{2}\right) \frac{1-\mathrm{e}^{-2 \mu \mathrm{t}}}{2 \mu}+\mathrm{l}_{6}\left(\mathrm{a}_{0} \mathrm{~d}_{0} \mathrm{~h}_{0}+\mathrm{b}_{0} \mathrm{c}_{0} \mathrm{~h}_{0}\right) \\
& \frac{1-\mathrm{e}^{-(\mu+v) \mathrm{t}}}{\mu+\mathrm{v}}+\mathrm{l}_{7} \mathrm{c}_{0} \mathrm{~d}_{0} \mathrm{~h}_{0} \frac{1-\mathrm{e}^{-(2 \mu+v-\lambda) \mathrm{t}}}{2 \mu+v-\lambda} \\
& \left.+\mathrm{l}_{8} \mathrm{~d}_{0} \mathrm{~h}_{0}^{2} \frac{1-\mathrm{e}^{-(\mu+2 v-\lambda) \mathrm{t}}}{\mu+2 v-\lambda}+\mathrm{l}_{9} \mathrm{~b}_{0} \mathrm{~h}_{0}^{2} \frac{1-\mathrm{e}^{-(\lambda+2 v) \mathrm{t}}}{\lambda+2 v}\right\}
\end{aligned}
$$

$$
\begin{aligned}
\mathrm{c}=\mathrm{c}_{0}+\varepsilon\left\{\mathrm{n}_{1} \mathrm{c}_{0}^{3} \frac{1-\mathrm{e}^{-2 \mu \mathrm{t}}}{2 \mu}+\mathrm{n}_{2} \mathrm{c}_{0}^{2} \mathrm{~d}_{0} \frac{1-\mathrm{e}^{-2 \mu \mathrm{t}}}{2 \mu}\right\} \\
\mathrm{d}=\mathrm{d}_{0}+\varepsilon \mathrm{mc}_{0}^{2} \mathrm{~d}_{0} \frac{1-\mathrm{e}^{-2 \mu \mathrm{t}}}{2 \mu} \\
\mathrm{h}=\mathrm{h}_{0}+\varepsilon\left\{\mathrm{s}_{1} \mathrm{a}_{0}^{2} \mathrm{~h}_{0} \frac{1-\mathrm{e}^{-2 \lambda \mathrm{t}}}{2 \lambda}+\mathrm{s}_{2} \mathrm{a}_{0} \mathrm{c}_{0} \mathrm{~h}_{0} \frac{1-\mathrm{e}^{-(\lambda+\mu) \mathrm{t}}}{\lambda+\mu}\right. \\
+\mathrm{s}_{3} \mathrm{a}_{0} \mathrm{~h}_{0}^{2} \frac{1-\mathrm{e}^{-(\lambda+v) \mathrm{t}}}{\lambda+\mathrm{v}}+\mathrm{s}_{4} \mathrm{c}_{0}^{2} \mathrm{~h}_{0} \frac{1-\mathrm{e}^{-2 \mu \mathrm{t}}}{2 \mu}+\mathrm{s}_{5} \mathrm{c}_{0} \mathrm{~h}_{0}^{2} \\
\frac{1-\mathrm{e}^{-(\mu+v) \mathrm{t}}}{\mu+\mathrm{v}}+\mathrm{s}_{6} \mathrm{~h}_{0}^{3} \frac{1-\mathrm{e}^{-2 v \mathrm{t}}}{2 v}+\mathrm{s}_{7} \mathrm{a}_{0} \mathrm{~b}_{0} \mathrm{~h}_{0} \frac{1-\mathrm{e}^{-2 \lambda \mathrm{t}}}{2 \lambda} \\
+\mathrm{s}_{8}\left(\mathrm{a}_{0} \mathrm{~d}_{0} \mathrm{~h}_{0}+\mathrm{b}_{0} \mathrm{c}_{0} \mathrm{~h}_{0}\right) \frac{1-\mathrm{e}^{-(\lambda+\mu) \mathrm{t}}}{\lambda+\mu}+\mathrm{s}_{9} \mathrm{c}_{0} \mathrm{~d}_{0} \mathrm{~h}_{0} \frac{1-\mathrm{e}^{-2 \mu \mathrm{t}}}{2 \mu} \\
\left.+\mathrm{s}_{10} \mathrm{~d}_{0} \mathrm{~h}_{0}^{2} \frac{1-\mathrm{e}^{-(\mu+v) \mathrm{t}}}{\mu+\mathrm{v}}+\mathrm{s}_{11} \mathrm{~b}_{0} \mathrm{~h}_{0}^{2} \frac{1-\mathrm{e}^{-(\lambda+v) \mathrm{t}}}{\lambda+v}\right\}
\end{aligned}
$$

Hence, we obtain the first approximate solution of the equation (13) as:

$$
x(t, \varepsilon)=(a+b t) e^{-\lambda t}+(c+d t) e^{-\mu t}+h e^{-v t}+\varepsilon u_{1}
$$

where $a, b, c, d$ and $h$ are given by the equations (32) and $\mathrm{u}_{1}$ is given by (30).

\section{Results and Discussion}

The perturbation solution is usually compared to the numerical solution to test the accuracy of the approximate solution obtained by a certain perturbation method. Therefore, we have first considered the eigenvalues $\lambda=0.75, \mu=0.1$ and $v=3.82$. We have computed $x(t, \varepsilon)$ using (33), in which $\mathrm{a}, \mathrm{b}, \mathrm{c}, \mathrm{d}$ and $\mathrm{h}$ are obtained from (32) and $\mathrm{u}_{1}$ is calculated from equation (30) together with initial conditions $\mathrm{a}_{0}=0.25, \quad \mathrm{~b}_{0}=0.1, \quad \mathrm{c}_{0}=0.4, \quad \mathrm{~d}_{0}=0.35$ and $\mathrm{h}_{0}=0.5$ when $\varepsilon=0.1$. The result obtained from (33) for various values of $t$, and the corresponding numerical solution obtained by a fourth order Runge-Kutta method is plotted in the Fig. 1.

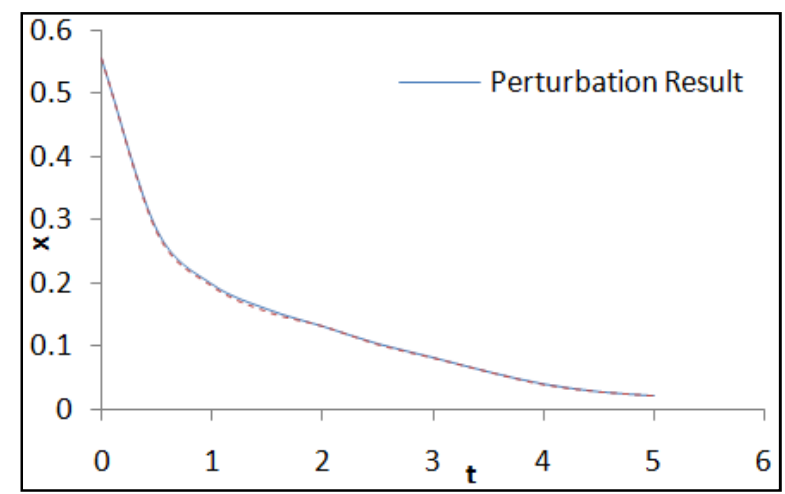

Fig. 1. Perturbation results are plotted by continuous line and numerical results are plotted by dotted line. 
Again, we have computed $\mathrm{x}(\mathrm{t}, \varepsilon)$ from (33) by considering values of $\lambda=0.70, \mu=0.1$ and $v=3.80$. We have computed $\mathrm{x}(\mathrm{t}, \varepsilon)$ using (33), in which $\mathrm{a}, \mathrm{b}, \mathrm{c}, \mathrm{d}$ and $\mathrm{h}$ are obtained from (32) and $u_{1}$ is calculated from equation (30) together with initial conditions $\mathrm{a}_{0}=0.35, \mathrm{~b}_{0}=0.15$, $\mathrm{c}_{0}=0.35, \mathrm{~d}_{0}=0.35$ and $\mathrm{h}_{0}=0.5$ when $\varepsilon=0.1$. The result obtained from (33) for various values of $t$, and the corresponding numerical solution obtained by a fourth order Runge-Kutta method is plotted in the Fig. 2.

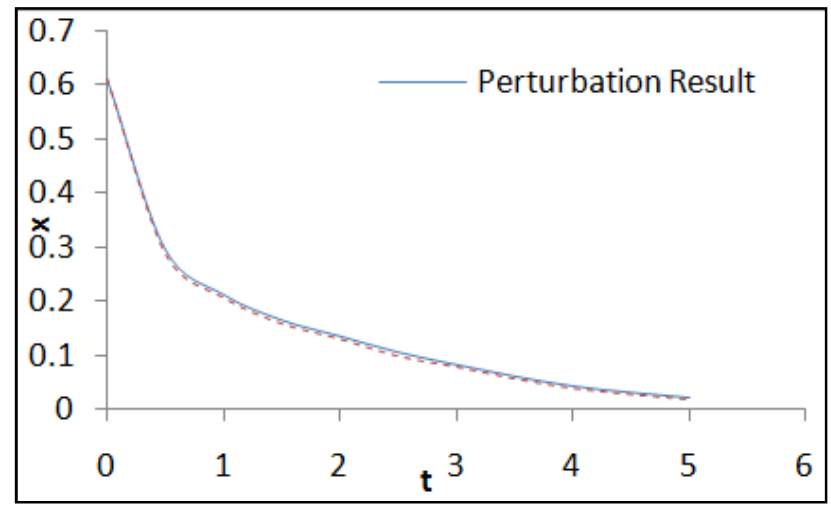

Fig. 2. Perturbation results are plotted by continuous line and numerical results are plotted by dotted line.

Finally, we have computed $x(t, \varepsilon)$ from (33) by considering values of $\lambda=0.69, \mu=0.15$ and $v=3.79$. We have computed $\mathrm{x}(\mathrm{t}, \varepsilon)$ using (33), in which $\mathrm{a}, \mathrm{b}, \mathrm{c}, \mathrm{d}$ and $\mathrm{h}$ are obtained from (32) and $u_{1}$ is calculated from equation (30) together with initial conditions $\mathrm{a}_{0}=0.45, \mathrm{~b}_{0}=0.20$, $\mathrm{c}_{0}=0.35, \mathrm{~d}_{0}=0.30$ and $\mathrm{h}_{0}=0.5$ when $\varepsilon=0.1$. The result obtained from (33) for various values of $t$, and the corresponding numerical solution obtained by a fourth order Runge-Kutta method is plotted in the Fig. 3.

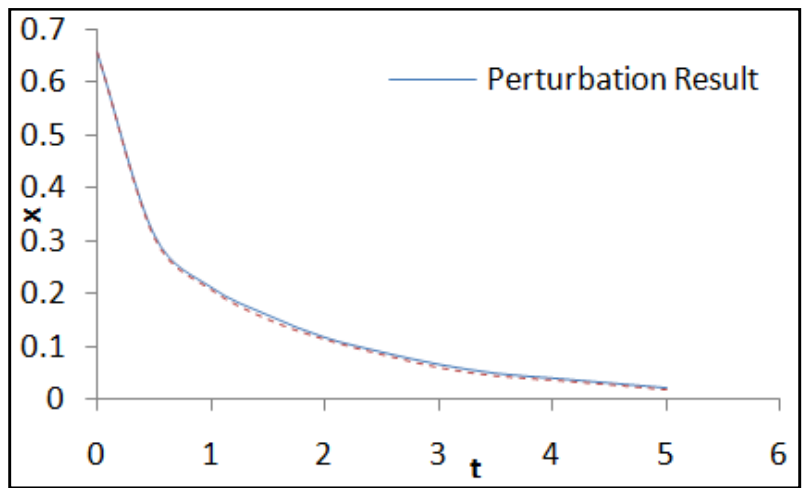

Fig. 3. Perturbation results are plotted by continuous line and numerical results are plotted by dotted line.

\section{Conclusion}

In conclusion, it is suggested that, in this article, the KBM method has been modified and applied successfully to the fifth order more critically damped nonlinear systems. In relation to the fifth order critically damped systems, the solutions are obtained in such circumstances where the four eigenvalues are pairwise equal and another eigenvalue is distinct. Normally, in the KBM method, it is noticed that much error occurs in the case of rapid changes of $x$ with respect to time $t$. However, it is suggested that all the aforementioned results obtained in this paper correspond accurately to the numerical solutions obtained by the fourth order Runge-Kutta method. It is, therefore, concluded that the modified KBM method provides highly accurate results, which can be applied for different kinds of nonlinear differential systems.

\section{Acknowledgement}

The authors are grateful to Mr. Md. Mizanur Rahman, Associate Professor, Department of Mathematics, Islamic University, Bangladesh, for his invaluable comments on the early draft of this paper. The authors are also thankful to Mr. Md. Imamunur Rahman for his assistance in editing this paper.

\section{References}

[1] Bogoliubov, N. N. and Mitropolskii, Y. A., Asymptotic Methods in the Theory of Nonlinear Oscillations, Gordan and Breach, New York, 1961.

[2] Krylov, N. N. and Bogoliubov, N. N., Introduction to Nonlinear Mechanics, Princeton University Press, New Jersey, 1947.

[3] Popov, I. P., "A Generalization of the Bogoliubov Asymptotic Method in the Theory of Nonlinear Oscillations (in Russian)", Dokl. Akad. USSR, vol. 3, pp. 308-310, 1956.

[4] Mendelson, K. S., "Perturbation Theory for Damped Nonlinear Oscillations", J. Math. Physics, vol. 2, pp. 3413$3415,1970$.

[5] Murty, I. S. N., “A Unified Krylov-Bogoliubov Method for Solving Second Order Nonlinear Systems", Int. J. Nonlinear Mech. vol. 6, pp. 45-53, 1971.

[6] Sattar, M. A., "An Asymptotic Method for Three-dimensional Over-damped Nonlinear Systems", Ganit, J. Bangladesh Math. Soc., vol. 13, pp. 1-8, 1993.

[7] Bojadziev, G. N., "Damped Nonlinear Oscillations Modeled by a 3-dimensional Differential System", Acta Mechanica, vol. 48, pp. 193-201, 1983.

[8] Shamsul, M. A. and Sattar, M. A., "An Asymptotic Method for Third Order Critically Damped Nonlinear Equations", J. Mathematical and Physical Sciences, vol. 30, pp. 291-298, 1996.

[9] Islam, M. R. and Akbar, M. A., "A New Asymptotic Solution for Third Order More Critically Damped Nonlinear Systems", IAENG Journal of Applied Mathematics, v. 39(1), 2009.

[10] Shamsul, M. A. and Sattar, M. A., "A Unified KrylovBogoliubov-Mitropolskii Method for Solving Third Order Nonlinear Systems", Indian J. pure appl. Math., vol. 28, pp. 151-167, 1997. 
[11] Akbar, M. A., Paul, A. C. and Sattar, M. A., "An Asymptotic Method of Krylov-Bogoliubov for Fourth Order Over-damped Nonlinear Systems", Ganit, J. Bangladesh Math. Soc., vol. 22, pp. 83-96, 2002.

[12] Islam, M. R., Uddin, M. S., Akbar, M. A., Huda, M. A. and Hossain, S. M. S., "A New Technique for Fourth Order Critically Damped Nonlinear Systems with Some Conditions", Bull. Cal. Math. Soc., vol. 100(5), pp. 501-514, 2008.

[13] Rahaman, M. M. and Rahman, M. M., "Analytical Approximate Solutions of Fifth Order More Critically Damped Systems in the case of Smaller Triply Repeated Roots", IOSR Journals of Mathematics, vol. 11(2), pp. 35-46, 2015 .

[14] Rahaman, M. M. and Kawser, M. A., "Asymptotic Solution of Fifth Order Critically Damped Non-linear Systems with Pair Wise Equal Eigenvalues and Another is Distinct.”, Journal of Research in Applied Mathematics, vol. 2(3), pp. 01-15, 2015.

[15] Shamsul, M. A., "Asymptotic Method for Certain Third-order Non-oscillatory Nonlinear Systems", J. Bangladesh Academy of Sciences, vol. 27, pp. 141-148, 2003.

[16] Sattar, M. A., “An asymptotic Method for Second Order Critically Damped Nonlinear Equations", J. Frank. Inst., vol. 321, pp. 109-113, 1986.

[17] Shamsul, M. A., "Asymptotic Methods for Second Order
Over-damped and Critically Damped Nonlinear Systems", Soochow Journal of Math., vol. 27, pp. 187-200, 2001.

[18] Shamsul, M. A., "A Unified Krylov-Bogoliubov-Mitropolskii Method for Solving $n$-th Order Nonlinear Systems”, J. Frank. Inst., vol. 339, pp. 239-248, 2002.

[19] Murty, I. S. N., "Deekshatulu, B. L. and Krishna, G., "On an Asymptotic Method of Krylov-Bogoliubov for Over-damped Nonlinear Systems”, J. Frank. Inst., vol. 288, pp. 49-65, 1969.

[20] Shamsul, M. A., "Bogoliubov's Method for Third Order Critically Damped Nonlinear Systems", Soochow J. Math., vol. 28, pp. 65-80, 2002.

[21] Shamsul, M. A., "Method of Solution to the n-th Order Overdamped Nonlinear Systems Under Some Special Conditions", Bull. Cal. Math. Soc., vol. 94, pp. 437-440, 2002.

[22] Shamsul, M. A., "On Some Special Conditions of Third Order Over-damped Nonlinear Systems", Indian J. Pure Appl. Math., vol. 33, pp. 727-742, 2002.

[23] Shamsul, M. A., "On Some Special Conditions of Overdamped Nonlinear Systems", Soochow J. Math., vol. 29, pp. 181-190, 2003.

[24] Murty, I. S. N., and Deekshatulu, B. L., "Method of Variation of Parameters for Over-Damped Nonlinear Systems", J. Control, vol. 9(3), pp. 259-266, 1969. 